Article

\title{
Quantum Game Beats Classical Odds-Thermodynamics Implications
}

\section{George Levy}

Entropic Power Corporation, 3980 Del Mar Meadows, San Diego, CA 92130, USA;

E-Mail: glevy@entropicpower.com; Tel.: +1-858-259-2226; Fax: +1-858-259-2233

Academic Editor: Antonio M. Scarfone

Received: 21 August 2015 / Accepted: 26 October 2015 / Published: 9 November 2015

\begin{abstract}
A quantum game is described making use of coins embodied as entangled Fermions in a potential energy well. It is shown that the odds are affected by the Pauli Exclusion Principle. They depend on the elevation in the energy well where the coins are selected, ranging from being a certainty of winning at the bottom of the well to being near classical at the top. These odds differ markedly from those in a classical game in which they are independent of elevation. The thermodynamics counterpart of the quantum game is discussed. It is shown that the temperature of a Maxwellian gas column in a potential energy gradient is independent of elevation. However, the temperature of a Fermion gas is shown to drop with elevation. The game and the gas column utilize the same components. When Fermions are used, a shifting of odds is produced in the game and a shifting of kinetic energy is produced in the thermodynamic experiment, leading to a spontaneous temperature gradient.
\end{abstract}

Keywords: quantum game; second law; entropy; Fermi-Dirac; Maxwell-Boltzmann; Fermion; Boson; temperature gradient; statistical mechanics; quantum mechanics

\section{Introduction}

The idea that a quantum game strategy can beat a classical one is not new. Flitney and Abbott [1] summarize a coin tossing game first described by Meyer [2] as follows:

Meyer considered the simple game "penny flip" that consists of the following: Alice prepares a coin in the heads state, Bob, without knowing the state of the coin, can choose 
to either flip the coin or leave its state unaltered, and Alice, without knowing Bob's action, can do likewise. Finally, Bob has a second turn at the coin. The coin is now examined and Bob wins if it shows heads. A classical coin clearly gives both players an equal probability of success unless they utilize knowledge of the other's psychological bias, and such knowledge is beyond analysis by standard game theory.

To quantize this game, we replace the coin by a two state quantum system such as a spin one-half particle. Now Bob is given the power to make quantum moves while Alice is restricted to classical ones. Can Bob profit from his increased strategic space? Let $\mid 0>$ represent the "heads" state and $\mid 1>$ the "tails" state. Alice initially prepares the system in the $\mid 0>$ state. Bob can proceed by first applying the Hadamard operator:

$$
H=\frac{1}{\sqrt{2}}\left(\begin{array}{cc}
1 & 1 \\
1 & -1
\end{array}\right)
$$

putting the system into the equal superposition of the two states: $\frac{1}{\sqrt{2}}(|0>+| 1>)$. Now Alice can leave the "coin" alone or interchange the states $\mid 0>$ and $\mid 1>$, but if we suppose this is done without causing the system to decohere either action will leave the system unaltered, a fact that can be exploited by Bob. In his second move he applies the Hadamard operator again resulting in the pure state $|0\rangle$ thus winning the game. Bob utilized a superposition of states and the increased latitude allowed him by the possibility of quantum operators to make Alice's strategy irrelevant, giving him a certainty of winning.

\section{-A.P. Flitney and D. Abbott}

In Meyer's game, the coin is manipulated sequentially by the players and the quantum player (Bob) utilizes the Hadamard operator to ensure that the outcome is always tail $\mid 0>$. Meyer does not describe a real world implementation for his game.

This paper describes a physical embodiment for a coin tossing game by representing a coin as a Fermion inside a potential energy well and shows that a player can manipulate the outcome of the game simply by changing her particle's elevation in the well. In addition, the thermodynamics implications of this game are also discussed in which the temperature of a non-Maxwellian gas is also shown to depend on elevation.

\section{Classical Coin Game Thought Experiment}

Consider the following classical game played by a player, say Alice, against the "House": The dealer tosses a large number of coins but their final states are hidden until the player selects two coins. Before uncovering them, she calls her bet: "matched" or "mismatched." as shown in Figure 1. Matching coin combinations include (Head, Head) and (Tail, Tail). Mismatching coins include (Head, Tail) or (Tail, Head). In a classical game the odds are obviously equal since $\mathrm{p}(\mathrm{Head}, \mathrm{Head})=\mathrm{p}(\mathrm{Tail}$, Tail $)=p($ Head, Tail $)=p($ Tail, Head $)=0.25$. The entropy corresponding to making a bet is:

$$
S=0.25 \log \left(\frac{1}{0.25}\right)=0.25 \log 4=0.25 \times 2 \log 2=0.5 \text { shannons }
$$


Playing such a game, Alice has a 50\% chance of winning. Can she improve her odds by using a quantum strategy?

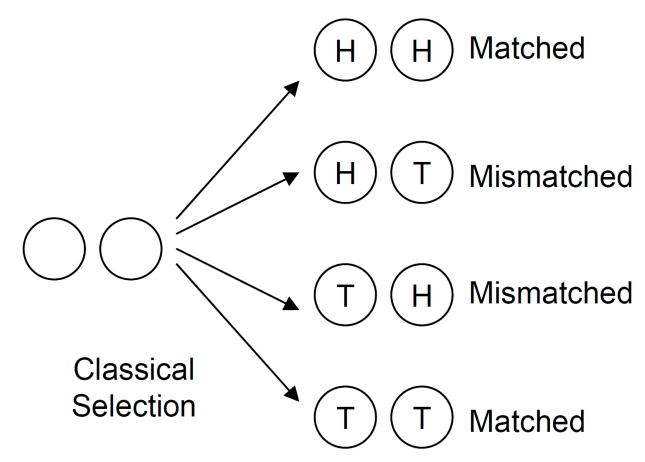

Figure 1. In a classical environment the selected coins are uncorrelated, and could be (Head, Head), (Head, Tail), (Tail, Head) or (Tail, Tail). Alice wins 50\% of the time.

\section{Quantum Coin Game Thought Experiment}

Let us assume that coins are actually Fermions with "Head" having, for example, a physical representation of spin up, and "Tail", of spin down. Furthermore, let's say that the coins are entangled in a potential energy well characterized by a number of discrete quantum states. In addition, let each coin possess energy, the lowest value being the ground state. This coin arrangement is exemplified by the well-known electronic configuration in an atom. To make it more interesting, we can assume that the atom is not in the ground state, so that some low energy levels are unoccupied. The general description of such system follows the Fermi-Dirac distribution.

Since the coins are Fermions, their states are constrained by the Pauli Exclusion Principle which requires that they are different as shown in Figure 2.

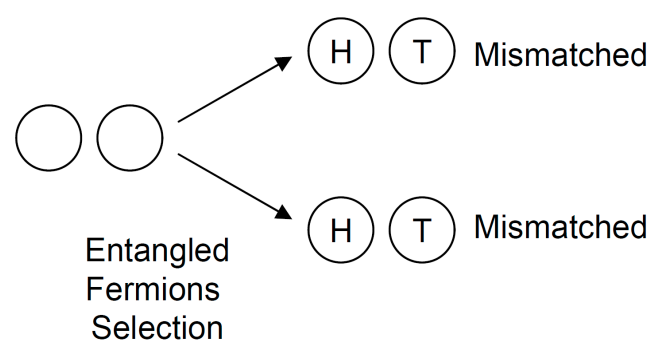

Figure 2. When the coins are Fermions in the ground state and constrained in a potential energy well, the Pauli Exclusion Principle requires them to be different allowing Alice to win all the time.

The lowest energy levels are the most crowded and include two anti-parallel states, both states being occupied, one coin being "Head" (spin up) and the other coin being "Tail" (spin down). For the sake of simplicity let us assume the ground state where the coins are perfectly entangled such that if both coins are selected from the ground state they form a mismatched pair.

At the top of the energy well, the states are less crowded than at the bottom and therefore it is possible to find unoccupied states, or coins occupying anti-parallel states as well as parallel states. For example 
two coins can be Head (spin up), or two coins can be "Tail" (spin down). For the sake of simplicity we shall also assume that a coin selected from the top level of the energy well is unentangled from the other coins such that when combined with another coin it would form a matched or mismatched pair with equal probability.

Therefore, Alice can increase her chances of winning, simply by selecting low energy coins and calling a "mismatch". Selecting from the bottom of the energy well, the entropy for each play is:

$$
S=1 \times \log (1)=0 \text { shannons }
$$

Note that all Alice needs to do is observe the coins, not remove them from the well.

\section{Discussion of the Coin Experiments}

One should note that if classical coins are used, the odds are even and independent of elevation. In his game, Meyer shifts the odds in favor of Bob by using a quantum coin being flipped repeatedly and applying the Hadamard operator to ensure that the outcome is always "Tail". In contrast, the game outlined above utilizes a multiplicity of quantum coins having an anti-symmetric wave function (Fermions). The coins are located in a potential energy well, thereby subjected to the Pauli Exclusion Principle. The player draws two coins, his goal being to get a mismatch. The odds are shifted in favor of Alice by selecting coins at a low elevation where the coins are entangled and have a higher probability for mismatch. The same conclusion would have been reached had the coins been Bosons and the winning combinations, matched pairs. The requirements for such a game are:

(1) Non-Maxwellian objects such as Fermions or Bosons.

(2) A potential energy gradient that crowds the objects and forces them into their characteristic non-Maxwellian distribution. For example, if the objects are Fermions, their distribution is, according to Landauer formalism, the product of their density of state and the Fermi-Dirac distribution.

The classical and quantum coin experiments have a Thermodynamics counterpart as explained below.

\section{Classical Gas Thought Experiment}

The coin thought experiment described above can be translated to the context of Thermodynamics. Consider a column of gas subjected to a force field and held in a container with perfectly reflecting and elastic walls, floor and ceiling. A game between Alice and the House is defined as follows. The House provides Alice with the position of each molecule and Alice selects two molecules, A and B. If A has more kinetic energy than B, Alice wins, otherwise the House wins. The question for Alice is whether she can improve her odds by selecting molecules at different elevation in the column.

One could rephrase the game in more friendly thermodynamics terms. Is the temperature at the top and at the bottom of the potential energy gradient the same or different?

One shall first consider the classical case shown in Figure 3, which uses a Maxwellian gas such as air, and a force such as gravity. 


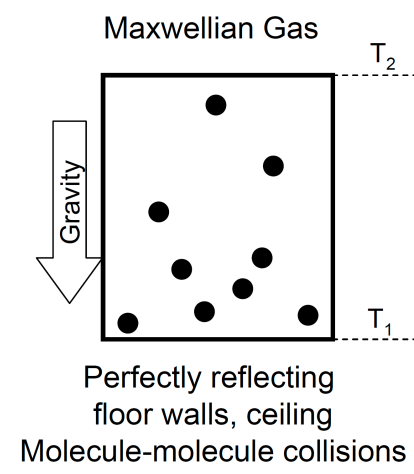

Figure 3. Classical gas subjected to a force field and enclosed in a perfectly reflecting box. Is the temperature of the gas the same or different at the top and at the bottom?

The molecules collide with each other thereby evolving a Maxwellian distribution. The distribution of the gas at ground level is given by the Maxwell-Boltzmann distribution equation:

$$
f_{M B}\left(E_{k}\right)=2\left(\frac{1}{k_{B} T}\right)^{3 / 2} \sqrt{\frac{E_{k}}{\pi}} \exp \left(\frac{-E_{k}}{k_{B} T}\right)
$$

also shown by the red curve at the bottom of Figure 4 (see Appendix A for details).

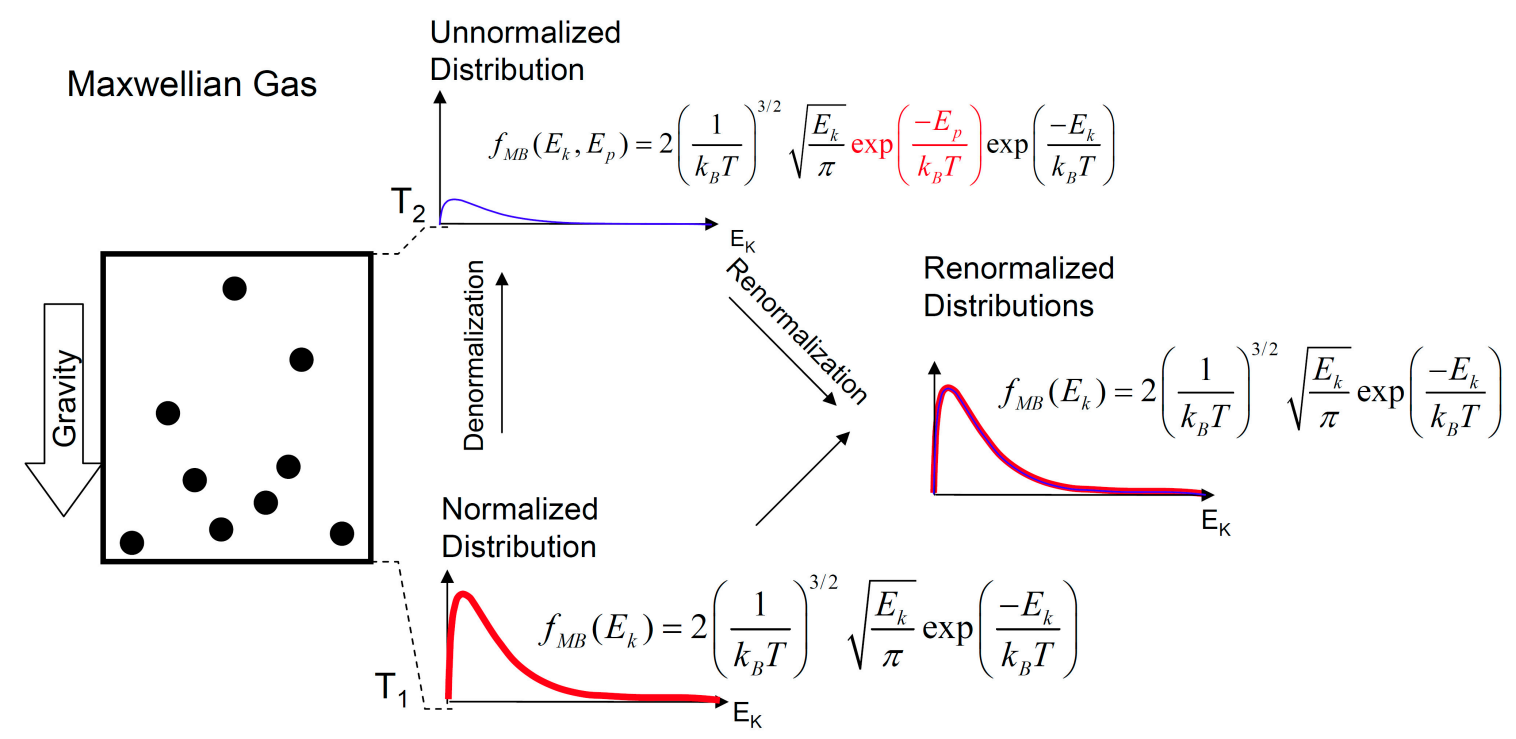

Figure 4. To compare the temperature of a classical gas at the bottom and at the top of a gas column, the following steps are taken: (1) a distribution is defined at the bottom; (2) The distribution at the top is obtained by inserting potential energy, thereby denormalizing the distribution; (3) The distribution at the top is then renormalized and compared to the one at the bottom. No shift in the first moment indicates that the gas is isothermal with elevation in conformance with the Second Law.

The equation for the distribution comprises three kinds of terms. The first corresponds to a normalization factor such that the area under the curve is unity. The second term is the square root of kinetic energy. The third term is the Maxwell term which is exponential with kinetic energy. The strict exponential nature of this term is important as shall be explained. 
The distribution at the top of the column can be generated by inserting potential energy $E_{p}$ in the exponential term adjacent to the kinetic energy $E_{k}$ to express the total energy of molecules:

$$
f_{M B}\left(E_{k}, E_{p}\right)_{\text {unnormalized }}=2\left(\frac{1}{k_{B} T}\right)^{3 / 2} \sqrt{\frac{E_{k}}{\pi}} \exp \left(\frac{-E_{k}-E_{p}}{k_{B} T}\right)
$$

Because it is expressed within an exponent, potential energy can also be factored out in its own exponential term as shown in the equation below:

$$
f_{M B}\left(E_{k}, E_{p}\right)_{\text {unnormalized }}=2\left(\frac{1}{k_{B} T}\right)^{3 / 2} \sqrt{\frac{E_{k}}{\pi}} \exp \left(\frac{-E_{k}}{k_{B} T}\right) \exp \left(\frac{-E_{p}}{k_{B} T}\right)
$$

One must note that inserting potential energy denormalizes the distribution, reducing its amplitude and resulting, as expected, in a lower density of molecules at higher elevation as shown at the top of Figure 4. Of concern however, is not density but temperature. A change in temperature can be expressed by a shift in the first moment of the distribution which can be visually expressed by comparing the distributions at the bottom and at the top. This comparison, however, must be made after the distribution at the top is renormalized. Since potential energy is expressed exponentially, it can be factored out as indicated in Equation (6) and eliminated by the renormalization process. The resulting renormalized equation for the gas at the top is:

$$
f_{M B}\left(E_{k}\right)_{\text {renormalized }}=2\left(\frac{1}{k_{B} T}\right)^{3 / 2} \sqrt{\frac{E_{k}}{\pi}} \exp \left(\frac{-E_{k}}{k_{B} T}\right)
$$

which is identical to the distribution at the bottom Equation (4). The corresponding curves, thick red for the bottom and thin blue for the top are shown at the right of Figure 4. These curves are identical, implying that the first moments of both curves are the same, and that the average kinetic energy and the temperature are invariant with elevation.

As expected, the Maxwellian gas is isothermal with elevation in compliance with the Second Law. This experiment corresponds to the coin selection experiment in which the player comes out even against the House (assuming no entrance or playing fee).

\section{Quantum Gas Thought Experiment}

The quantum version of the gas experiment outline above requires that the molecules be non-Maxwellian, for example Fermions. As shown in Figure 5, these can be embodied in a thermoelectric material by electrons or holes which can be regarded as being in a gas phase (see Appendix B for details).

The thermoelectric slab is subjected to an electric field, produced for example by insulated capacitor plates or a junction. The slab is composed of a material with a high ZT, such that the carriers do not collide with heat phonons. ZT is a dimensionless figure of merit used in the thermoelectric industry. It is a measure of the ability of a given material to produce thermoelectric power and is given by $Z T=\frac{\sigma S^{2} T}{\lambda}$. It depends on the electrical conductivity $\sigma$, the Seebeck coefficient $S$, the temperature $T$ and the thermal conductivity $\lambda$. 


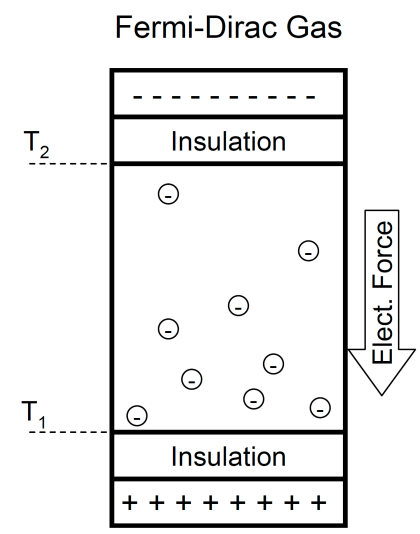

Very Hi ZT Thermoelectric Material;

No Phonon-Electron Scattering

High Electron-Electron Scattering

Figure 5. A gas comprised of quantum particles (e.g., Fermions) is subjected to a force field and enclosed in a slab of semiconductor. Is the temperature of the gas the same or different at the top and at the bottom?

Because of the high ZT, electrons are mostly ballistic with respect to the lattice, but they still interact between themselves thereby evolving their own Fermion distribution. A detailed description of the experiment is given by the author in [3] and experimental evidence is provided that the phenomenon described below does occur.

In this experiment as in the previous one, the question being asked is whether the temperature at the top and at the bottom of the potential energy gradient is the same or different. The same procedure can be applied as before. First the distribution at the bottom of the potential energy gradient is defined. In accordance with Landauer-Boltzmann formalism, since the gas is composed of Fermions, their distribution is the product of the density of state (which we will assume to be parabolic for the sake of simplicity):

$$
g_{c}\left(E_{t}\right)=\frac{8 \pi \sqrt{2}}{h^{3}} m^{3 / 2} \sqrt{E_{k}}
$$

and of the Fermi-Dirac distribution:

$$
F_{F D}\left(E_{t}\right)=\frac{1}{1+\exp \left(\frac{E_{k}+E_{c}-E_{F}}{k_{B} T}\right)}
$$

Combining Equations (8) and (9), and normalizing yields the normalized Fermion distribution:

$$
f_{F D}\left(E_{k}\right)=A_{1} \sqrt{E_{k}} \frac{1}{1+\exp \left(\frac{E_{k}+E_{c}-E_{F}}{k_{B} T}\right)}
$$

where $A_{1}$ is the normalization factor.

The distribution at the top of the slab is obtained by inserting potential energy $E_{p}$ next to kinetic energy $E_{k}$ in the Fermi-Dirac term, to express the total energy of molecules: 


$$
f_{F D}\left(E_{k}\right)=A_{1} \sqrt{E_{k}} \frac{1}{1+\exp \left(\frac{E_{k}+E_{p}+E_{c}-E_{F}}{k_{B} T}\right)}
$$

The step of inserting potential energy denormalizes the distribution which is shown as the thin blue curve at the top of Figure 6.

A change in temperature corresponds to a change in the first moment of the distribution. The change can be visually determined by comparing the original distribution shown at the bottom of Figure 6 with the distribution at the top. However, this comparison must be made after the distribution at the top is renormalized.

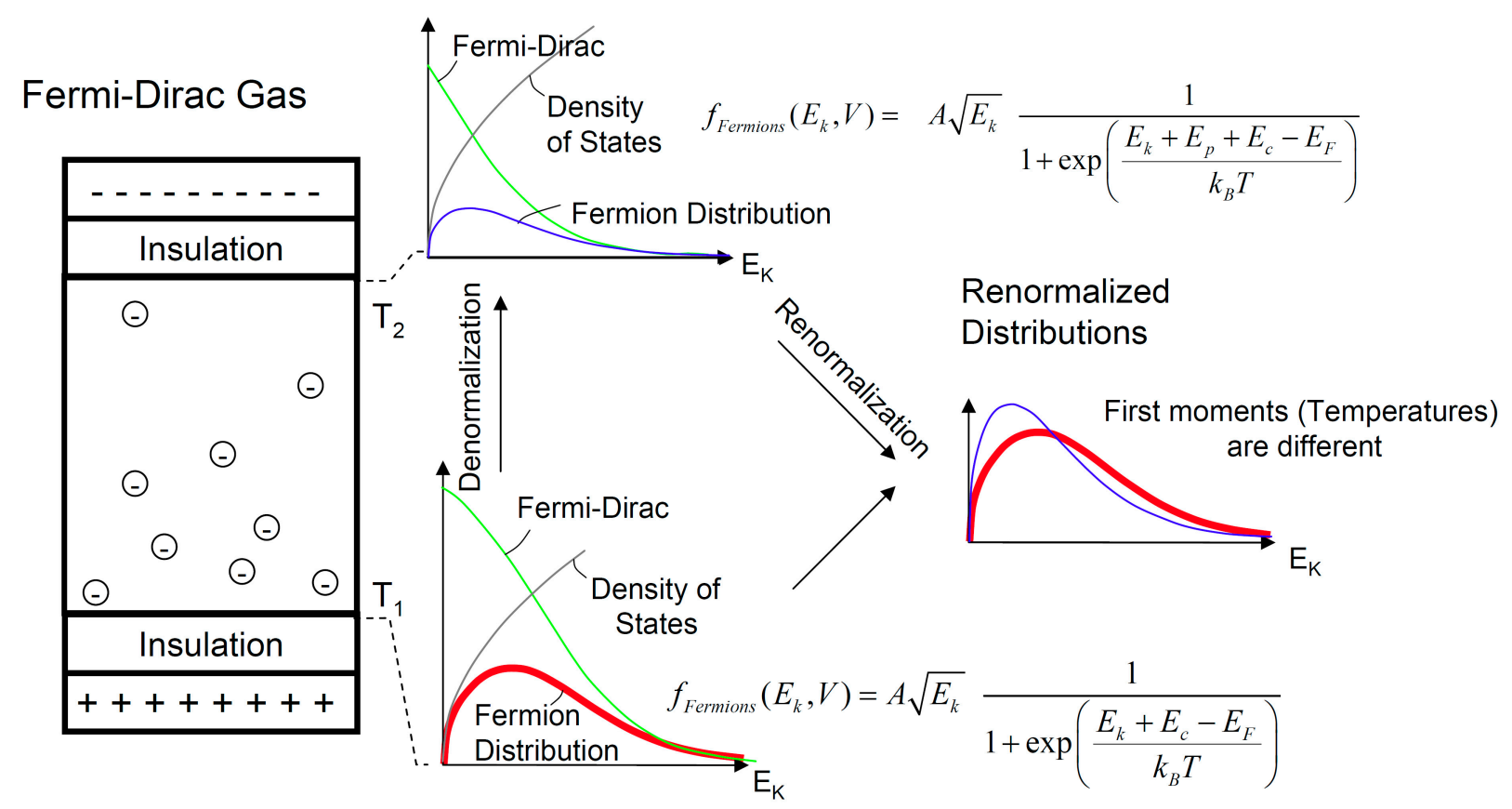

Figure 6. As in the classical experiment, the temperatures of the gas at the bottom and at the top of a potential energy gradient are compared using the following procedure: (1) a distribution is generated at the bottom of the column (2) The distribution at the top is obtained by inserting potential energy, thereby denormalizing the distribution; (3) The distribution at the top is renormalized and compared to the one at the bottom. A shift in the first moment indicates that the gas is colder at the top of the columns than at the bottom.

$$
f_{F D}\left(E_{k}\right)_{\text {renormalized }}=A_{2} \sqrt{E_{k}} \frac{1}{1+\exp \left(\frac{E_{k}+E_{p}+E_{c}-E_{F}}{k_{B} T}\right)}
$$

where $A_{2}$ is the normalization factor. In contrast to the previous experiment, the distribution is not a strict exponential function of potential energy. Therefore, potential energy cannot be eliminated by the renormalization process, resulting in the distribution at the top being skewed toward the left in comparison with the one at the bottom and indicating a shift of the first moment. This shift demonstrates a lower average kinetic energy, and therefore a lower temperature with elevation. The 
reason for this result is the non-strictly exponential nature of the Fermi-Dirac distribution which prevents the cancellation of potential energy by renormalization.

Figure 7 summarizes the result of the classical and quantum gas experiment. It shows the energy distribution of the gas at two elevations (thick red: ground level; thin blue: elevation above ground) for a Maxwellian gas (a) and for a non-Maxwellian gas (b). The calculator software for generating these curves is available at [4].

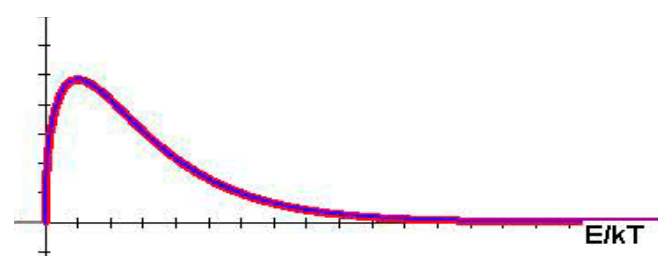

(a)

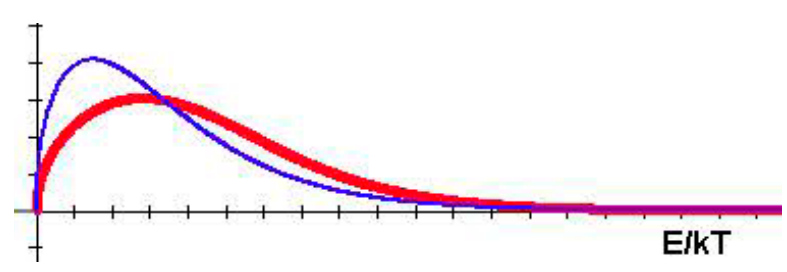

(b)

Figure 7. (a) Normalized energy distribution for a Maxwellian gas at two elevations, (red curve for ground level and blue curve for elevation above ground). The two distributions are superimposed indicating that their first moment is identical, and that the gas is isothermal with elevation. These distributions were calculated using the Maxwell-Boltzmann distribution; (b) Normalized energy distribution for a Fermion gas at two elevations, (red curve for ground level and blue curve for elevation above ground). The distribution above ground is skewed to the left compared to the one at ground level, indicating a smaller first moment and a drop in temperature with elevation. These distributions were calculated as the product of a parabolic density of state and the Fermi-Dirac distribution.

\section{Discussion of the Gas Experiments}

The thermodynamics counterpart of the quantum game above is fully described by the author in [3] and reproduced in part in this paper. He shows that when a gas comprised of non-Maxwellian particles (Fermions or Bosons) is subjected to an external force (potential energy gradient), the temperature of the gas drops with elevation. This thermodynamic effect is Quantic in nature and can be used to play the Quantum game described above.

In contrast, when the gas is Maxwellian, elevation has no effect on temperature - the gas is isothermal in accordance with the Second Law. This corresponds to the classical game described above in which Alice's chances of winning are $50 \%$ - not including any fees she may have to pay the "House" for playing the game, which would reduce her expected winnings.

\section{Conclusions}

A coin tossing game is described in which a player plays against the House by selecting two coins. Before uncovering them, she calls a "match" or a "mismatch". The classical game utilizes coins that follow rules of Classical Physics. When the player plays classically, she comes out even.

The quantum game uses coins embodied as Fermions and subjected to a potential energy gradient in a potential well. It is shown that a quantum strategy can beat classical odds. According to the Pauli Exclusion Principle no two Fermions can occupy the same state and therefore, selecting Fermions from 
the bottom of the energy well is the best strategy to achieve a mismatch. The odds of winning the game are shown to depend on the elevation of the Fermions being selected ranging from being a certainty of winning at the bottom of the well to being near classical at the top. These odds differ markedly from those in a classical game in which they are independent of elevation.

The thermodynamics counterpart of the quantum game is discussed in which a Maxwellian gas and a non-Maxwellian gas are subjected to a force. Temperature is shown to be independent of elevation for the Maxwellian gas in accordance with the Second Law, but drops with elevation for the non-Maxwellian gas.

The game and the Fermion gas column utilize the same components: Fermions are subjected to a force field, and develop their own characteristic distribution. A shifting of odds is produced in the game and a shifting of kinetic energy is produced in the thermodynamic experiment: the Fermion gas column develops a spontaneous temperature gradient.

Obviously such a phenomenon is not allowed in classical Physics but is permitted in Quantum Mechanics when the particles follow a non-Maxwellian distribution.

The three laws of Thermodynamics can facetiously be described as "You can't win", "You can't get even", and "You can't get out of the game". The game is rigged by the rules of Classical Mechanics. The best strategy then, is to play according to different rules, in other words, to cheat. Quantum Mechanics allows you to do just that.

\section{Conflicts of Interest}

The author declares no conflicts of interest.

\section{Appendix A. Derivation of Maxwell-Boltzmann Distribution in the Presence of an External Force Field}

The first step is to express the Maxwell-Boltzmann distribution when an external force field is applied on the gas. In the absence of a force each component of the velocity vector has a normal distribution:

$$
\begin{aligned}
& f_{M B}\left(v_{x}\right)=\left(\frac{m}{2 \pi k_{B} T}\right)^{1 / 2} \exp \left(\frac{-m v_{x}^{2}}{2 k_{B} T}\right) \\
& f_{M B}\left(v_{y}\right)=\left(\frac{m}{2 \pi k_{B} T}\right)^{1 / 2} \exp \left(\frac{-m v_{y}^{2}}{2 k_{B} T}\right) \\
& f_{M B}\left(v_{z}\right)=\left(\frac{m}{2 \pi k_{B} T}\right)^{1 / 2} \exp \left(\frac{-m v_{z}^{2}}{2 k_{B} T}\right)
\end{aligned}
$$

If a force is applied in the $z$ direction the gas acquires potential energy $E_{p}=m g z$ and Equation (15) becomes:

$$
f_{M B}\left(v_{z}, z\right)=\left(\frac{m}{2 \pi k_{B} T}\right)^{1 / 2} \exp \left(\frac{-\frac{1}{2} m v_{z}^{2}-m g z}{k_{B} T}\right)
$$


The probability distribution of the vector $\bar{v}$ at a given elevation $z$ can be seen as the joint probability of three independent probabilities $f_{M B}\left(v_{x}\right), f_{M B}\left(v_{y}\right)$ and $f_{M B}\left(v_{z}, z\right)$ along each degree of freedom. Hence:

$$
f_{M B}(\bar{v}, z)=f_{M B}\left(v_{x}, v_{y}, v_{z}, z\right)=f_{M B}\left(v_{x}\right) f_{M B}\left(v_{y}\right) f_{M B}\left(v_{z}, z\right)
$$

Therefore, substituting Equations (13), (14) and (16) into Equation (17) produces:

$$
f_{M B}(\bar{v}, z) d v_{x} d v_{y} d v_{z}=\left(\frac{m}{2 \pi k_{B} T}\right)^{3 / 2} \exp \left(\frac{-m\left(v_{x}^{2}+v_{y}^{2}+v_{z}^{2}\right)}{2 k_{B} T}\right) \exp \left(\frac{-m g z}{k_{B} T}\right) d v_{x} d v_{y} d v_{z}
$$

To obtain the distribution for the kinetic energy $E_{k}$, the velocity components are expressed in spherical coordinates. A velocity volume element is given by:

$$
d v_{x} d v_{y} d v_{z}=v^{2} \sin \theta d \theta d \phi d v
$$

Integrating $\theta$ and $\phi$, this volume element becomes:

$$
d v_{x} d v_{y} d v_{z}=v^{2} d v \int_{0}^{2 \pi} d \phi \int_{0}^{\pi} \sin \theta d \theta=4 \pi v^{2} d v
$$

Substituting Equation (20) into Equation (18) produces:

$$
f_{M B}(v, z) d v=\left(\frac{m}{2 \pi k_{B} T}\right)^{3 / 2} \exp \left(\frac{-m v^{2}}{2 k_{B} T}\right) \exp \left(\frac{-m g z}{k_{B} T}\right) 4 \pi v^{2} d v
$$

Since $d E_{k}=m v d v=\sqrt{2 m E_{k}} d v$ and defining the potential energy as $E_{p}=m g z$ results in:

$$
f_{M B}\left(E_{k}, E_{p}\right)=2\left(\frac{1}{k_{B} T}\right)^{3 / 2} \sqrt{\frac{E_{k}}{\pi}} \exp \left(\frac{-E_{k}}{k_{B} T}\right) \exp \left(\frac{-E_{p}}{k_{B} T}\right)
$$

Normalizing Equation (22) yields:

$$
f_{M B}\left(E_{k}, E_{p}\right)_{\text {normalized }}=\frac{2\left(\frac{1}{k_{B} T}\right)^{3 / 2} \sqrt{\frac{E_{k}}{\pi}} \exp \left(\frac{-E_{k}}{k_{B} T}\right) \exp \left(\frac{-E_{p}}{k_{B} T}\right)}{\int_{0}^{\infty} 2\left(\frac{1}{k_{B} T}\right)^{3 / 2} \sqrt{\frac{E_{k}}{\pi}} \exp \left(\frac{-E_{k}}{k_{B} T}\right) \exp \left(\frac{-E_{p}}{k_{B} T}\right) d E_{k}}
$$

Rearranging yields:

$$
f_{M B}\left(E_{k}, E_{p}\right)_{\text {normalized }}=\frac{2\left(\frac{1}{k_{B} T}\right)^{3 / 2} \sqrt{\frac{E_{k}}{\pi}} \exp \left(\frac{-E_{k}}{k_{B} T}\right) \exp \left(\frac{-E_{p}}{k_{B} T}\right)}{\exp \left(\frac{-E_{p}}{k_{B} T}\right) \int_{0}^{\infty} 2\left(\frac{1}{k_{B} T}\right)^{3 / 2} \sqrt{\frac{E_{k}}{\pi}} \exp \left(\frac{-E_{k}}{k_{B} T}\right) d E_{k}}
$$

Since $\int_{0}^{\infty} 2\left(\frac{1}{k_{B} T}\right)^{3 / 2} \sqrt{\frac{E_{k}}{\pi}} \exp \left(\frac{-E_{k}}{k_{B} T}\right) d E_{k}=1$, we get: 


$$
f_{M B}\left(E_{k}, E_{p}\right)_{\text {normalized }}=2\left(\frac{1}{k_{B} T}\right)^{3 / 2} \sqrt{\frac{E_{k}}{\pi}} \exp \left(\frac{-E_{k}}{k_{B} T}\right)
$$

Hence the distribution is independent of potential energy $E_{p}$ and of elevation in compliance with the Second Law as shown in the Figure $7 \mathrm{a}$.

\section{Appendix B. Derivation of the Fermion Distribution in the Presence of an External Force Field}

Consider a Fermion gas embodied as electrons confined to an n-doped semiconductor slab as shown in Figure 5. The thermally significant electrons are those that happen to be in the conduction band and that are made available by the Fermi-Dirac distribution. Such electrons must have a positive kinetic energy $E_{k}>0$ and a total energy $E_{t}$ greater than the potential energy of the band's bottom $E_{c}$, i.e., $E_{t}>E_{c}$. In the conduction band, the electron's density of states is given by [1,2] by:

$$
g_{c}\left(E_{t}\right)=\frac{8 \pi \sqrt{2}}{h^{3}} m^{3 / 2} \sqrt{E_{t}-E_{c}}
$$

Expressing kinetic energy as $E_{k}=E_{t}-E_{c}$ and substituting into Equation (26) produces:

$$
g_{c}\left(E_{t}\right)=\frac{8 \pi \sqrt{2}}{h^{3}} m^{3 / 2} \sqrt{E_{k}}
$$

The Fermi-Dirac distribution of the carriers can be expressed as:

$$
F_{F D}\left(E_{t}\right)=\frac{1}{1+\exp \left(\frac{E_{t}-E_{F}}{k_{B} T}\right)}
$$

and using $E_{k}=E_{t}-E_{c}$ we get:

$$
F_{F D}\left(E_{k}\right)=\frac{1}{1+\exp \left(\frac{E_{k}+E_{c}-E_{F}}{k_{B} T}\right)}
$$

In the presence of an electrical field, an electrical potential energy $E_{p}$ is induced in the carriers, and the Fermi-Dirac distribution is modified accordingly:

$$
F_{F D}\left(E_{k}, E_{p}\right)=\frac{1}{1+\exp \left(\frac{E_{k}+E_{c}+E_{p}-E_{F}}{k_{B} T}\right)}
$$

In accordance with Landauer-Boltzmann formalism, the electron density $n\left(E_{t}, E_{p}\right)$ is the product of the carrier density of states and the Fermi distribution:

$$
f_{\text {Fermions }}\left(E_{k}, E_{p}\right)=n\left(E_{k}, E_{p}\right)=g_{c}\left(E_{k}\right) F_{F D}\left(E_{k}, E_{p}\right)
$$

Substituting Equations (27) and (30) into Equation (31) yields: 


$$
f_{\text {Fermions }}\left(E_{k}, E_{p}\right)=\frac{8 \pi \sqrt{2}}{h^{3}} m^{3 / 2} \sqrt{E_{k}} \frac{1}{1+\exp \left(\frac{E_{k}+E_{p}+E_{c}-E_{F}}{k_{B} T}\right)}
$$

Normalizing yields:

$$
f_{\text {Fermions }}\left(E_{k}, E_{p}\right)_{\text {normalized }}=\frac{\frac{8 \pi \sqrt{2}}{h^{3}} m^{3 / 2} \sqrt{E_{k}} \frac{1}{1+\exp \left(\frac{E_{k}+E_{p}+E_{c}-E_{F}}{k_{B} T}\right)}}{\int_{0}^{\infty} \frac{8 \pi \sqrt{2}}{h^{3}} m^{3 / 2} \sqrt{E_{k}} \frac{1}{1+\exp \left(\frac{E_{k}+E_{p}+E_{c}-E_{F}}{k_{B} T}\right)} d E_{k}}
$$

and cancelling constant terms produces:

$$
f_{\text {Fermions }}\left(E_{k}, E_{p}\right)_{\text {normalized }}=\frac{\sqrt{E_{k}} \frac{1}{1+\exp \left(\frac{E_{k}+E_{c}+E_{p}-E_{F}}{k_{B} T}\right)}}{\int_{0}^{\infty} \sqrt{E_{k}} \frac{1}{1+\exp \left(\frac{E_{k}+E_{c}+E_{p}-E_{F}}{k_{B} T}\right)} d E_{k}}
$$

Please note that, unlike in the Maxwell-Boltzmann case (Appendix A), the potential energy term $E_{p}$ is not readily cancelled causing the Fermion distribution with $E_{p}>0$ not to be identical with the distribution with $E_{p}=0$. Since the areas under the distributions are equal to unity, and the distribution are not congruent, the distributions must be skewed with respect to each other as shown in Figure $7 \mathrm{~b}$. This figure has been generated using a calculator program. It shows that the distributions have different first moments implying different temperatures, in violation of Clausius' formulation of the Second Law.

\section{References}

1. Flitney, A.P.; Abbott, D. An introduction to quantum game theory. Fluct. Noise Lett. 2002, 2, doi:10.1142/S0219477502000981.

2. Meyer, D.A. Quantum Strategies. Phys. Rev. Lett. 1999, 82, doi:10.1103/PhysRevLett.82.1052.

3. Levy, G.S. Anomalous Temperature Gradient in Non-Maxwellian Gases. In Ceramics for Energy Conversion, Storage, and Distribution Systems, Proceedings of the 11th International Conference on Ceramic Materials \& Components for Energy \& Environmental Applications, Vancouver, BC, Canada, 14-19 June 2015; Wiley: Hoboken, NJ, USA, 2016; Volume 255, in press.

4. Levy, G.S. Non-Maxwellian Distribution Calculator. Available online: http://www.entropicpower.com/ onMaxwellianCalculatorSetup.zip?attredirects $=0 \& d=1$ (accessed on 28 October 2015).

(C) 2015 by the authors; licensee MDPI, Basel, Switzerland. This article is an open access article distributed under the terms and conditions of the Creative Commons Attribution license (http://creativecommons.org/licenses/by/4.0/). 